\title{
A Graph Theory Framework for Analysis of Forest Connectivity and Important of Individual Forest Patch in Pennar River Basin of India
}

\section{Pradeep Kumar Rajput *}

Center for Study of Regional Planning and Economic Growth, Barkatullah University, Bhopal, MP, India

${ }^{*}$ Corresponding author: Rajput PK, Center for study of Regional Planning and Economic Growth, Barkatullah University, Bhopal, MP, India, Tel: +91-8517885747; Email: pkrseven@gmail.com

Rec date: April 17, 2018; Acc date: April 30, 2018; Pub date: May 02, 2018

Copyright: ( 2018 Rajput PK, et al. This is an open-access article distributed under the terms of the Creative Commons Attribution License, which permits unrestricted use, distribution, and reproduction in any medium, provided the original author and source are credited.

\begin{abstract}
Graph theory based forest connectivity in pennar river basin in India. Connectivity is important for exchange their genetic material from one forest patch to another forest patch for regulates the ecosystem and maintain the biodiversity level both flora and fauna in particular region. On the earth everything is connected directly or indirectly. A land scape level forest connectivity to regulate the biodiversity, wildlife movement, seed dispersal and ecological factor. In this paper we analysis of forest patch connectivity between one forest patch to rest of other forest patches in pennar river basin. The study analyzed forest patches in 2005 is 1870,1995 is 2602,1985 is 2493 which is distributed in landscape area is $30532 \mathrm{~km}^{2}, 26889 \mathrm{~km}^{2}, 26951 \mathrm{~km}^{2}$. The study identify in different year (2005, 1995, $1985)$ only one components are important for connectivity $(6,20,20)$ it has $715,1525,1406$ number of patches and the total area of the components is $22449,19701,19640 \mathrm{in} \mathrm{km}^{2}$ on the basis of forest patch with decades changes the forest patches will be deceases form 1985 to 2005 . Conefor sensinode software used for quantified for landscape connectivity indices. The Conefor sensinode software performing two type of modeling one is binary connection and probalistic connection. In this paper used binary connection model for landscape connectivity. For quantify the landscape connectivity, decide a threshold distance such as $100 \mathrm{~m}, 200 \mathrm{~m}, 250 \mathrm{~m}, 500 \mathrm{~m}, 750 \mathrm{~m}, 1000$ m, 2000 m, 3000 m, 4000 m, 5000 m, 7500 m, 10000 m, 15000 m, 2000 m, 25000 m. Graph theoretic indices used for landscape modeling they are IIC Integral index connectivity, H Harary, LCP landscape coincidences probability. To identify the important forest patch for conservation planning and wildlife management for the development in future.
\end{abstract}

Keywords: Forest; Graph theory; Landscape; Ecology

\section{Introduction}

Graph theory is a mathematical concept based on finite set of nodes and links. This concept was introduced by haray in 1969 [1]. Graph theory applied in a variety of discipline including ecology [2]. Bunn et al. demonstrated the first application of graph theory in simulating connectivity habitat network which result in suitable scenario for conservation biology [3]. Graph theory become a effective way of modeling habitats and ecological interactions among them [4-6]. Graph based modeling is a rapid tool in conservation assessment [5] and is not data demanding [7]. A graph or network is a set of nodes and edges, where nodes are single elements within the network and edges represent connectivity between nodes (above figure 1). Such that links connect two nodes. Also depend on the patch distance between the patches nodes represent the patches of suitable habitat surrounded by inhospitable habitat. The existence of a link between each pair of patches implies the potential ability between two patches, which are considered, connected. The set nodes is called component in graph theory. Landscape can viewed as a network of habitat patches connected by dispersing individual [3] Network topology is especially interesting because it is an emergent Property that affects qualities such as spread of information and diseases, vulnerability to disturbance and stability [8-10].

Although graph theory is newcomer in landscape ecology it has been widely use in various other discipline such as natural science and social science, where resulting models are graphs or network. Graph has been used to represent spatial relationship among the habitat patches [5] and among individuals on landscape [11]. For focal species. Graph have also been used to model of connectivity among habitat reserve, allow to assessment of conservation strategies for multiple species [12]. A most distinct use of graph theory is to produce raster model of landscape where connectivity is examined at the scale of a single raster cell [13-16]. These approaches are unified in their use of graph theory to represent connectivity of landscape.

Here we focus only one from of landscape graph; graph that model the relationships among patches of habitat. Defined for a forest area that is suitable for animals, birds and seed dispersal and exchange of genetic material are distinguished from matrix and serve as node (also called vertices) the connection among nodes, called links (also called edges) suggest that potential for movement or dispersal of focal specie. In the most common application of patches based graph, links represent the geographic distance between nodes and nodes are connected by links only when this distance when is below some ecological relevant movement threshold for the organism. Group of connected nodes are called components, and these imply that an organism inhabitant any node within the component can potentially move or disperse to any other nodes in the sane component. Node that have no links to other nodes are also considered to be component

In most case patches graph based model for functional connectivity because their links represent a functional response of the organism to the landscape, that is links are not interpreted as structural features of the landscape or as corridors bur rather than as representing the connection among the patches. 


\section{Study area}

Pennar river basin is one the major east flowing river basin of India. It is situated between $77^{\circ} \mathrm{E}$ to $81^{\circ} \mathrm{E}$ longitudes and $11^{\circ} \mathrm{N}$ to $16.5^{\circ} \mathrm{N}$ latitudes. The main sub basins of pennar basin are pennar, palar, kunderu and paleru. The total catchment area is $14,3700 \mathrm{~km}^{2}$. Most of it area lie in Tamil nadu and Andhra Pradesh states of India. Pennar is a river of southern India. The Pennar rises on the hill of Nandi Hills in Chikballapur District of Karnataka state, and runs north and east through the state of Andhra Pradesh to empty into the Bay of Bengal area. The river basin receives $500 \mathrm{~mm}$ average rain fall annually. The river basin lies in the rain shadow region of Eastern Ghats.

\section{Vegetation}

The upper basin was formerly covered by tropical dry forest, thorn forest, and xeric shrub lands. Most of the dry tropical forest has now disappeared, due to clearance for grazing and overharvesting the forests for timber and firewood, replaced by thorny scrublands. The remnant forests of the Deccan are largely deciduous, dropping their leaves in the dry winter and spring months. The East Deccan dry evergreen forests of Coastal Andhra were evergreen, but these forests have largely been reduced to tiny remnant pockets.

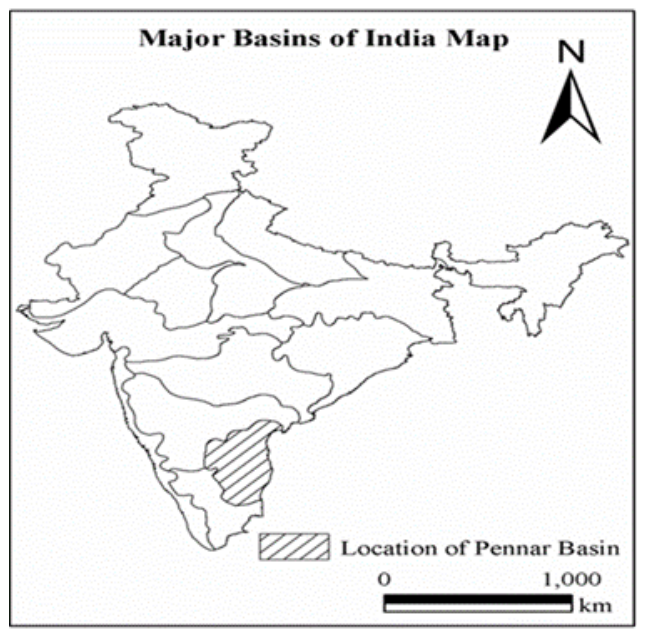

Figure 1: Study area map.

\section{Materials and Methods}

We focus on graph perspective and conceive the landscape as asset of habitat patches (nodes) and connecting elements (links). A links defined as an element that comprise no habitat area but represent the possibility of dispersal between two habitat patches, A links may correspond to physical corridor or it may symbolize the potential of an organism to directly disperse between two habitat patches through favorable land cover. A landscape that contains habitat area is considered a habitat patch, even though its main role may be serve as a stepping stone or connecting elements between to other habitat area.

A habitat patch i is here characterized by an attribute value (ai) typically habitat area, quality weighted habitat area [17], habitat suitability, core area, area to the power of coefficient that typically ranges from 0.1 to 0.5 [18], probability of occurrence [19], population size or another attribute relevant for analysis.
The strength of each link is characterized by pij which is the probability of direct dispersal between patches $i$ and $j$ (without passing nay other intermediate habitat patch) within a given time (e.g., one generation). Values of pi may be quantified using a variety of input data and method depending on the availability of data and the objectives and scale of the analysis. These include simle Euclidean distance [5], effective (least cost) distance, spatially explicit dispersal models $[19,20]$, or actual movement data derived from radio tracking or mark release recapture experiments When performing connectivity analysis two different connection models are possible [21]. In the present study we use binary (graph with unweight links) connection models.

\section{Graph theoretic indices}

There are large number of graph theoretic analysis technique and indices and during the past decade, many have been applied and new have been developed for analysis of landscape connectivity. In this paper we use Conefor sensinode software for quantified the landscape connectivity indices, The Conefor sensinode Software is performing a two different connection models one is binary connection and probalistic connection. Here we use only binary connection model for landscape connectivity in pennar river basin.

The integral index connectivity IIC described in pascal-hortal and Saura is based on binary connection model (it consider each two habitat or nodes as either connected or not, with no intermediate modulation of the strength or frequency of use of the connection between them) and given by

$$
I I C=\frac{\sum_{i=1}^{n} \sum_{j=1}^{n} \frac{a_{i} \cdot a_{j}}{1+n l_{i j}}}{A_{L}^{2}}
$$

Where $\mathrm{n}$ is the total number of nodes in the landscape ai and ai are the attributed of nodes I and $\mathrm{j}$ nlij is the number of links in the shortest path (topological distance) between nodes I and j; AL is the maximum landscape attributed (e.g., if the attributed is the area, then $\mathrm{AL}$ corresponding to the total study area, including both habitat and nonhabitat patches). If the value of $\mathrm{AL}$ is not specified, the IIC numerous values can be instead of IIC.

The IIC includes the intra flux and connector as describe by sauranadrubio, these fraction will be automatically calculated if selected IIC, one the three dIIc fraction estimating the amount of dispersal fluxes between a particular patch (as the orgi or destination of those fluxes) and the rest of the patches in the landscape, while dIIc connector fraction measuring the contribution of the analyzed patch to the connectivity between other patches as a connecting elements or stepping stone between them and the dIICintra is the contribution of patch is involved in the intra-patch connectivity within in components, dIIC intra is fully independent of how patch may be connected to other patches does not depend on the dispersal distance of the focal species and intra patch is completely isolated.

NL-Number of links: As a landscape is more connected it will present a large total number of links (connection between habitat nodes in the landscape).

NC-Number of component: A component is a set of nodes in which path exists between every pair of nodes an isolate node or patches make itself up a component. As the landscape more connected it will fewer component. 


$$
\begin{aligned}
& \text { H- Haray: } \\
& H=\frac{1}{2} \sum_{i=1}^{n} \sum_{j \neq i \neq j}^{n} \frac{1}{n l_{i j}}
\end{aligned}
$$

Where $\mathrm{n}$ is the total number of node in the landscape and nlij is the number of links in the shortest paths between patches I and $j$ (shortest path in terms of topological distance). For patches are not connected (belong to different component) $n \mathrm{lij}=\infty$. note that the case $\mathrm{i}=\mathrm{j}$ is not in the sum for $\mathrm{H}$. As a landscape is more connected it will present a higher $\mathrm{H}$ value.

LCP Landscape coincidence probability: LCP range from 0 to 1 increase with improved connectivity as in computed.

$$
L C P=\sum_{i=1}^{n}\left(\frac{c_{i}}{A_{L}}\right)
$$

Where NC is the number of components in the landscape, ci is the total component attribute (sum of the attributes of all the nodes belonging to that component) and $\mathrm{AL}$ is the maximum landscape attribute. If the node attribute is area (habitat patch area) then AL is the total landscape area (area of the analyzed region, comprising both habitat and non-habitat patches) and LCP $=1$ when all the landscape is occupied by habitat. CCP and LCP are generalizations of the degree of coherence [22] by considering components instead of individual patches.

\section{Material used}

Three different year (1985 to 2005) satellite data were used for prepared forest type polygon

- 1985 IRS Landsat MSS.

- 1995 IRS LISS I.

- 2005 IRS LISS III.

\section{Software used}

- Arc GIS 9.10.

- Conefor sensinode 2.6.

\section{Conefor sensinode a software package for analyzing landscape connectivity}

Confer Sensinode is a software for quantifying the importance of habitat patches for maintaining landscape connectivity through spatial graphs and habitat availability (reachability) metrics [23]. Conefor is conceived as a tool for decision making support and landscape planning and habitat conservation. Conefor incorporates nine graph based connectivity metrics among which, the IIC [24] and PC [21].

Conefor quantifies connectivity from a functional perspective as the inputs for Conefor consist both in information about the spatial structure and configuration of the habitat in the landscape and the dispersal capabilities of the organism under analysis and if required behavioral response to the spatial heterogeneity of the landscape. The most recent compilation is a new Conefor 2.6 beta version, which has been used for the analysis presented in this dissertation. This new version 2.6 includes new methodological developments related to the habitat availability metrics and extends the importance analysis to individual links and connector, among other improvements (Figure 2).

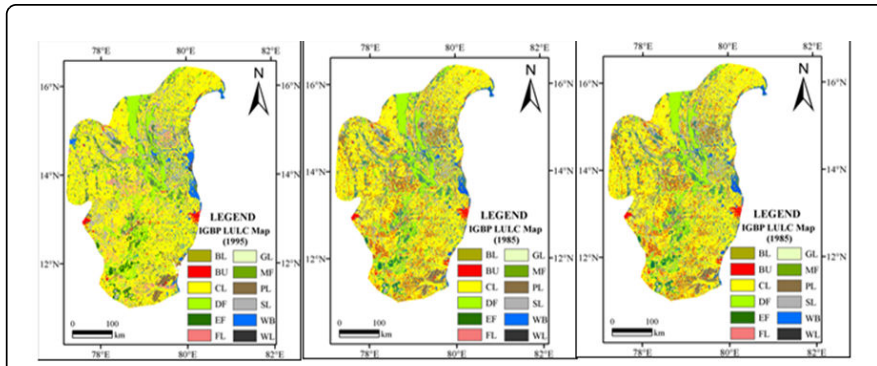

Figure 2: Land use and land cover map from 2005, 1995, 1985.

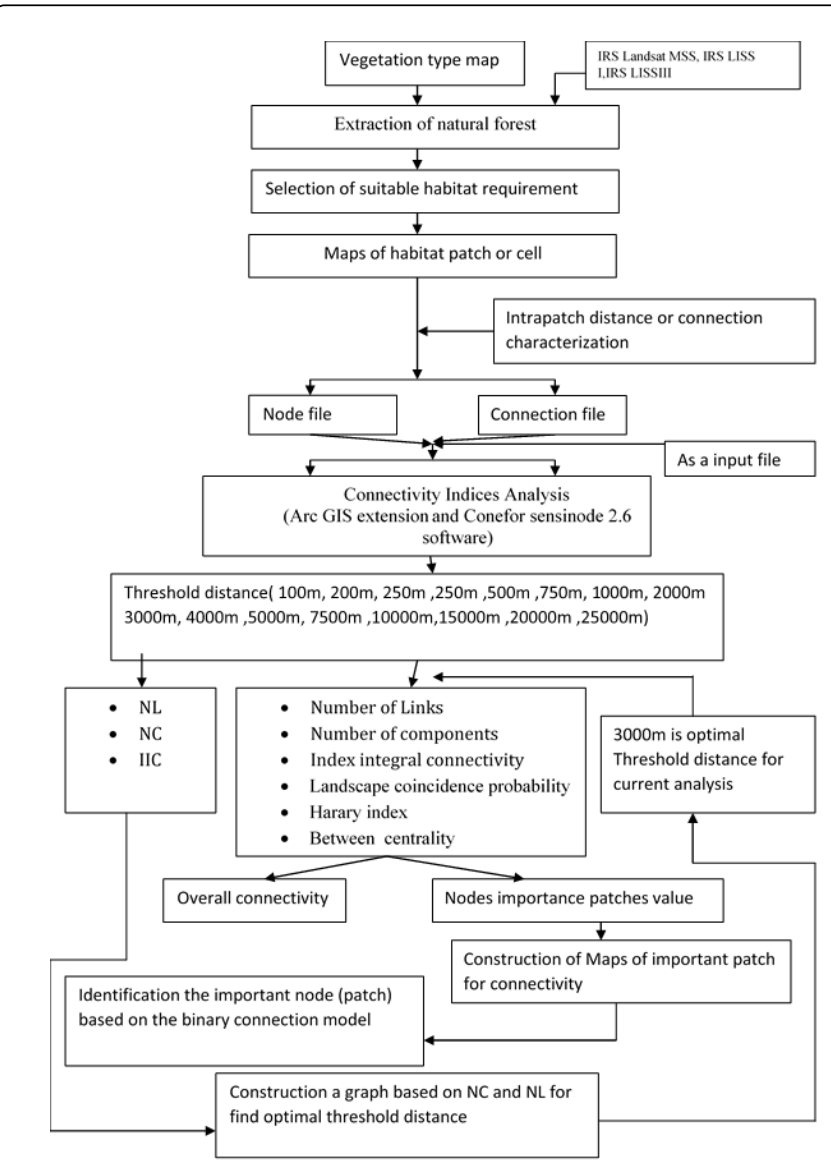

Figure 3: Flow chat of Methodology.

Despite its recent development, Conefor is having a rapid acceptance and it has been applied in wide variety of conservation plans and scientific studies as shown in http://www.conefor.org/ application.html. The most recent compilation is new Conefor 2.6 beta version, which has been used for the analysis presented in this study new version, includes new Methodical developments related to the habitat availability metrics and extends the importance to analysis to individual links and connectors among other improvements (see www.conefor.org or further details and update) (Figure 3). 


\section{Threshold distance of landscape graphs}

As the maximum inter patch dispersal distance increase was increases, the forest cover map become increasingly connected and eventually coalesced into a single, large graph spanning the entire habitat distribution. At a $100 \mathrm{~m}$ to $1000 \mathrm{~m}$ threshold distance, the landscape was largely composed of independent patches and small habitat clusters. For organism capable of dispersing $100 \mathrm{~m}$ to $1000 \mathrm{~m}$ the landscape was highly fragmented. At $1000 \mathrm{~m}$ to $7500 \mathrm{~m}$ large sub graph formed but the landscape was still divided into several habitat clusters and above $7500 \mathrm{~m}$ to $25000 \mathrm{~m}$, most of the habitat distribution was connected. Although most of the habitat was joined at $25 \mathrm{~km}$, only a single edge existed between the large sub graphs in the pennar river basin in Andhra Pradesh of the habitat distribution. The vertices at either end of single connecting edges are known as articulation points because removing either one would bisect the graph [1]. At a threshold distance of $25000 \mathrm{~m}$, the graph was highly interconnected and in general way many alternate pathways from from any one patch to another. In 2005 all the nodes (forest patches) was connected to made a one component. While in 1985 and 1995 all the nodes was connected to make a two component.

\section{Results and Discussion}

\section{Analysis for optimal threshold distance based on the number of links and number of components}

A range of thershold distance is $100 \mathrm{~m}$ to $25000 \mathrm{~m}(100,200,250$, $500,750,1000,2000,3000,4000,5000,7500,10000,15000,20000$, $25000 \mathrm{~m}$ ). Number of links increases linearly at the shortest thershold distance and becomes saturated as the thershold distance is increases. And the number of components become decreases at the high thershold distance $(25000 \mathrm{~m})$ due to this all nodes (forest patches) are connected to made a one components. NL is less and NC is high at lower thershold distance. If the NC increase, connectivity among them should happen apart from within the components. The number of component was found to decrease with increase thershold distance. Hence choosing the thershold distance based on the highest NC is essential. In the pennar river basin, forest distribution at $3000 \mathrm{~m}$ thershold gave 213 components in 1985, 215 components in 1995, 280 components in 2005 it is a favorable for the fragmentation analysis it did not give irregular distribution, all small fragmented nodes make a component and become interconnected to each other. Number of patches in 2005 is 1870,1995 is 2602,1985 is 2493 on the basis of forest patch with decades changes the forest patches will be deceases.

Numbers of isolated patches are less in $3000 \mathrm{~m}$ thershold distance it was observed that thershold distance more than $3000 \mathrm{~m}$ yield all the nodes (forest patches) make into one components it not suitable for the current study. And those less than $3000 \mathrm{~m}$ yield patch distribution with more NC and irregular fragmented patches given rise to many components and isolated components which is not suitable for analyses, if more than $3000 \mathrm{~m}$ yield patch distribution with less NC and ultimately all nodes make a single component. Hence the optimal thershold distance is $3000 \mathrm{~m}$.

We study the connectivity of forest patch through landscape graph indices, a binary connection model consider each two node as either connected or not, with no intermediate of the connection strength or dispersal feasibility among them. A link between two node is typically in the model by comparing the distance between them and specified thershold distance for the organism and species under study but here only make a model on the basis of different graph theory indices (NL, NC, H, BC, LCP, IIC) fewer change were detected by simple binary indices $(\mathrm{NL}, \mathrm{NC}, \mathrm{H})$ and important indices for connectivity is dIIC and dLCP it define the connectivity to each nodes while BC is measure connectivity for single node.

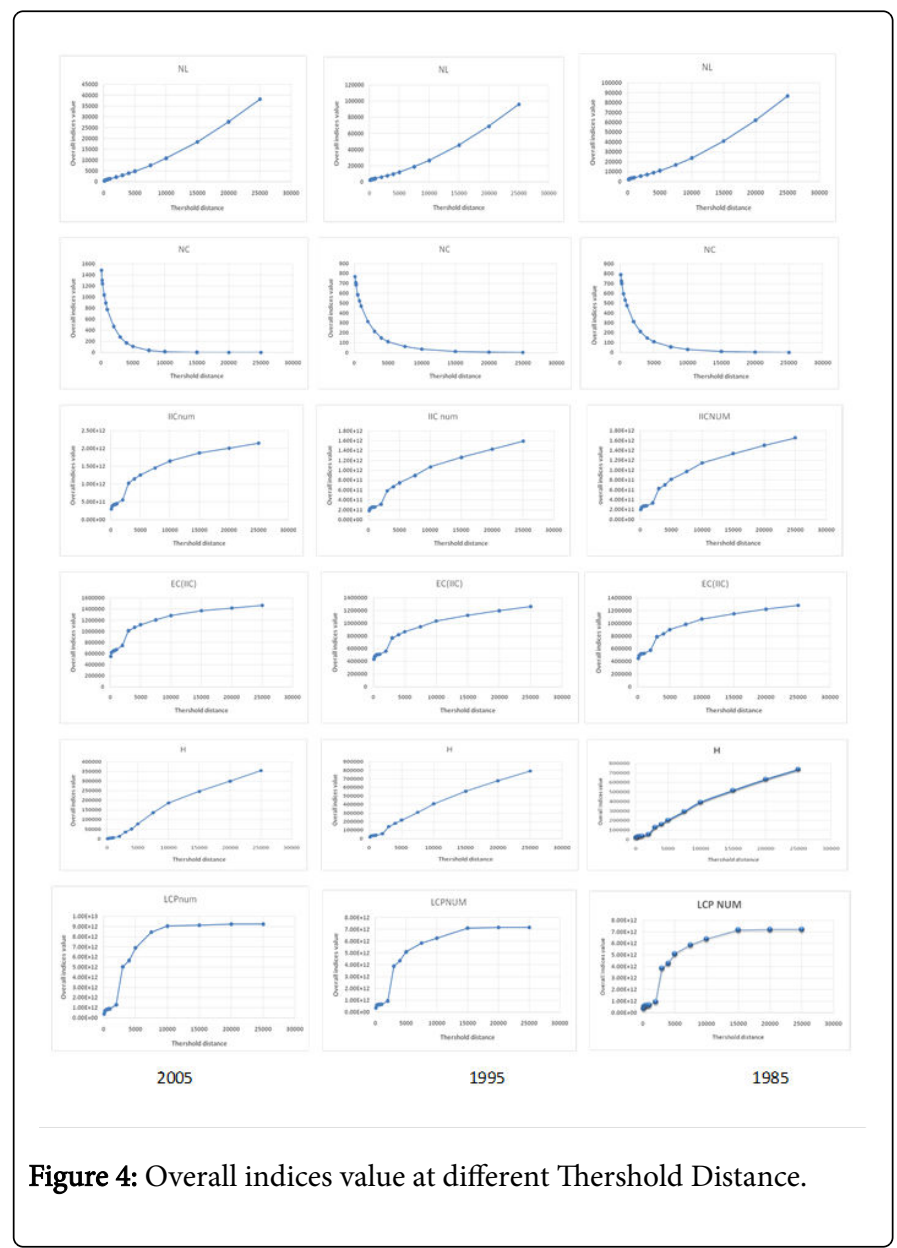

\section{Graph metrics related to the top 10 components}

In $2005,1995,1985(280,215,213)$ components were obtained for thershold distance $3000 \mathrm{~m}$. Figueres 4-6 shows the number of patches and area distribution in each component. In the top 10 components having a highest number of patches and remaining components contributed very low percentage of patches to the total connectivity of the landscape. Among the top 10 components 6, 20, 20 (2005, 1995, 1985) components showed suitable requirement for connectivity. These components cover center part of the study area for forest patch connectivity. At optimal thershold distance, choosing the components also depend on the numbers of patch, area dA, dIIC and dLCP. Components 6, 20, 20 got the highest dA, dIIC, dLCP, BC and $\mathrm{H}$ value. But only a single patch itself got the highest value and all the remaining components got very low value of graph theory indices. In different year $(2005,1995,1985)$ only one components are important for connectivity $(6,20,20)$ it has $715,1525,1406$ number of patches and the total area of the components is $22449,19701,19640$ in $\mathrm{km}^{2}$. The total area of all nodes which is present in landscape is 30532, 26889, 26951 in $\mathrm{km}^{2}$. Here distribution of the nodes (forest patches) is important for connectivity to each patch is significant and suitable for fragmentation studies. Thus components 6,20 and 20 are the optimal 
Citation: Rajput PK (2018) A Graph Theory Framework for Analysis of Forest Connectivity and Important of Individual Forest Patch in Pennar

Page 5 of 9

component with reference to patch distribution and nodes importance value (dA, dIIC, BC, H, dLCP). Hence components 6 and 20 are chosen as optimal group of patch for connectivity.

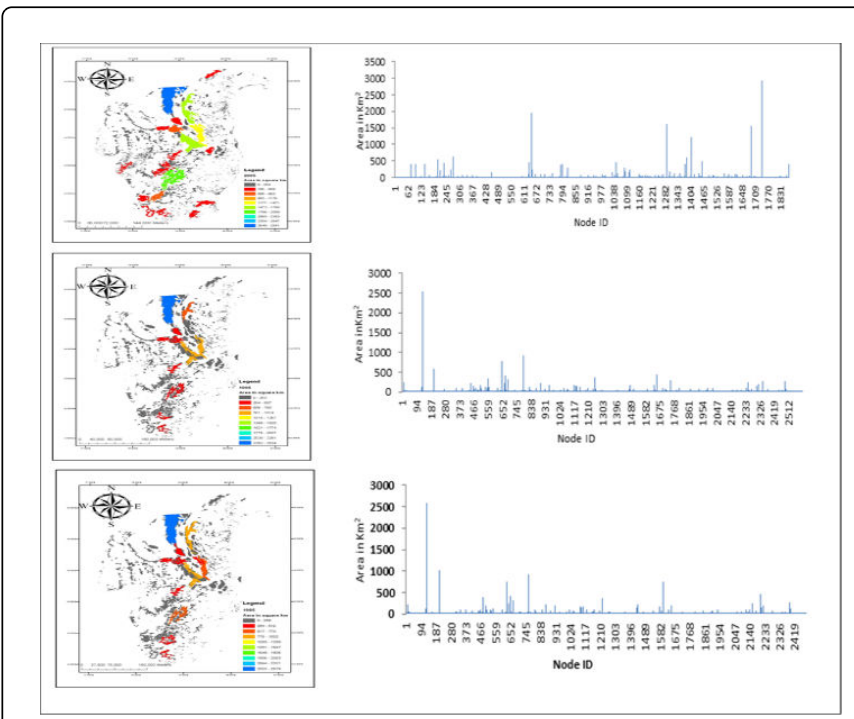

Figure 5: Classified map of area distribution and graph.

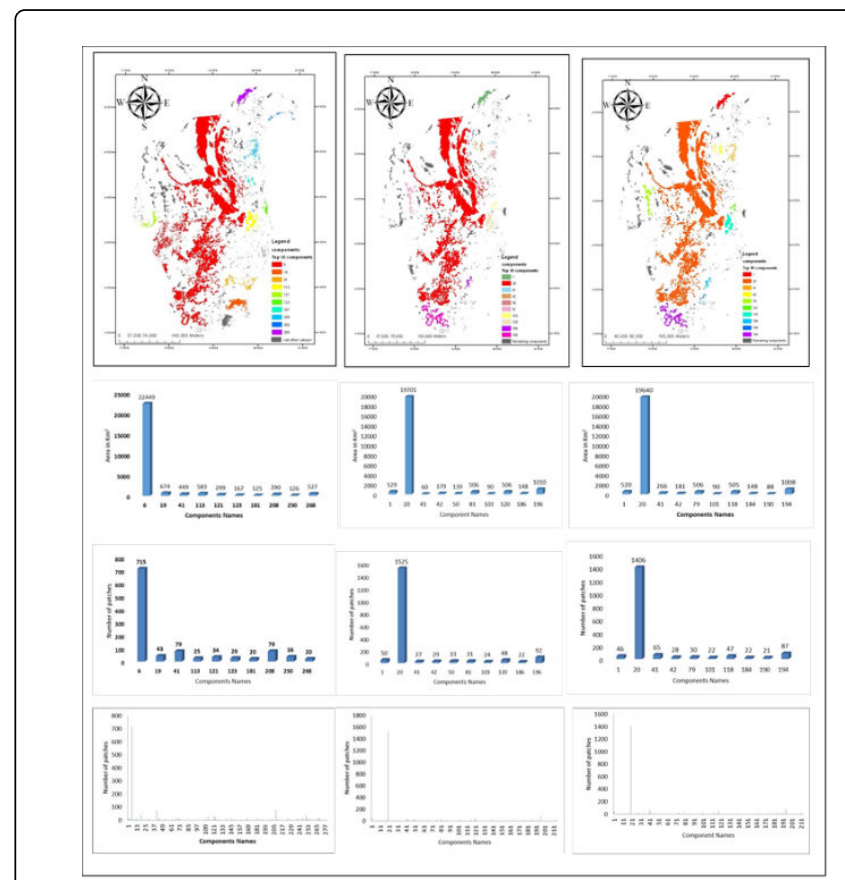

Figure 6: Classified map of area distribution and graph.

\section{Importance of individual habitat patches for landscape connectivity in 2005}

Above figures shows nodes important with reference to area dIIC for all the node ID in component 6 at the threshold distance $3000 \mathrm{~m}$. Components 6 has 715 patches (nodes) with the total area is 22449 in square kilometer. The main focus of study to find out the most important forest patches to participate in connectivity of nodes for movement of animals and seed dispersal and high importance value indices. Here consider patches of high importance value will facilities the understanding of patches significant for connectivity. Hence the top 20 patches (nodes)having a high value dIIC, dIIC (intra flux, connector) $\mathrm{dLCP}, \mathrm{BC}, \mathrm{dH}$ value analyzed as shown in Figures 7-10. This approach helped to study the concentration of dIIC value at different intervals around 20 patches are getting higher than 1 dIIC value. All the remaining patches are in range $0-1$. Those patches having very big area has a high dIIC value. Remaining patches are less than 0.05 because there area is very less.

Node ID (patches) namely 649, 1289 have the highest dIIC, dLCP and ALCP value while other node ID 649, 1742 for dA and dIICintra, Node ID 649, 786 for dNL, Node ID 1049, 1289 for BC having the highest value. For connecting these two very high important value patches choosing suitable patches with high to medium importance value required. Fraction of all indices result are shown in figure. Node ID 1289, 649, 1385, 1406, 1742 got a highest dIIC value; Node ID 1742, $649,1289,1692,1406$ got a highest dIICintra value and dIICflux; Node ID 1289, 1385, 1403, 1507, 1049 got a highest dIIC connector value. Node ID 1289, 649, 1406, 786, 1049 got a highest haray value: Node ID $1049,1289,821,749,649$ got a highest BC value it measure connectivity for single node; Node ID 1289, 1385, 649, 1406, 1456 got a highest dLCP value; Node ID 786, 649, 1406, 202, 749, a got a highest dNL value; 1742, 649, 1289, 1692, 1406 got a highest dA value. Node Id 649,1289 has got the highest value in all binary indices value.

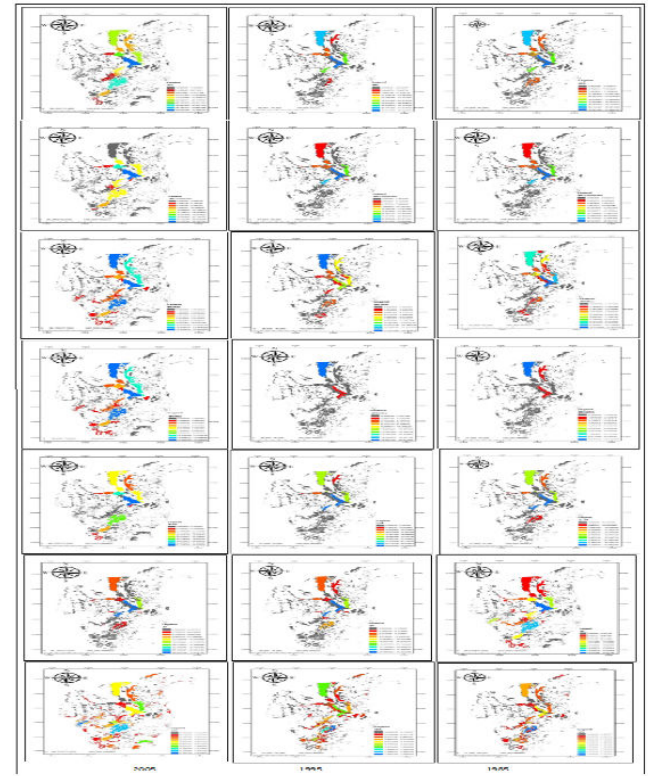

Figure 7: Classified map of all nodes value of graph theory indices.

These two nodes are very important role in connectivity in study area and remaining nodes are also participating in connectivity at 3000 $\mathrm{m}$ thershold distance. But they come under top 5 patches. Analysis the area of to dIIC and dLCP, those nodes has a big area got highest value of indices. Node Id 1742 got low value as compared to top 5 node but has a big area (294066 hacate) because connectivity depends on the location of node in the area, 1742 node is conner node is not good for connectivity. 


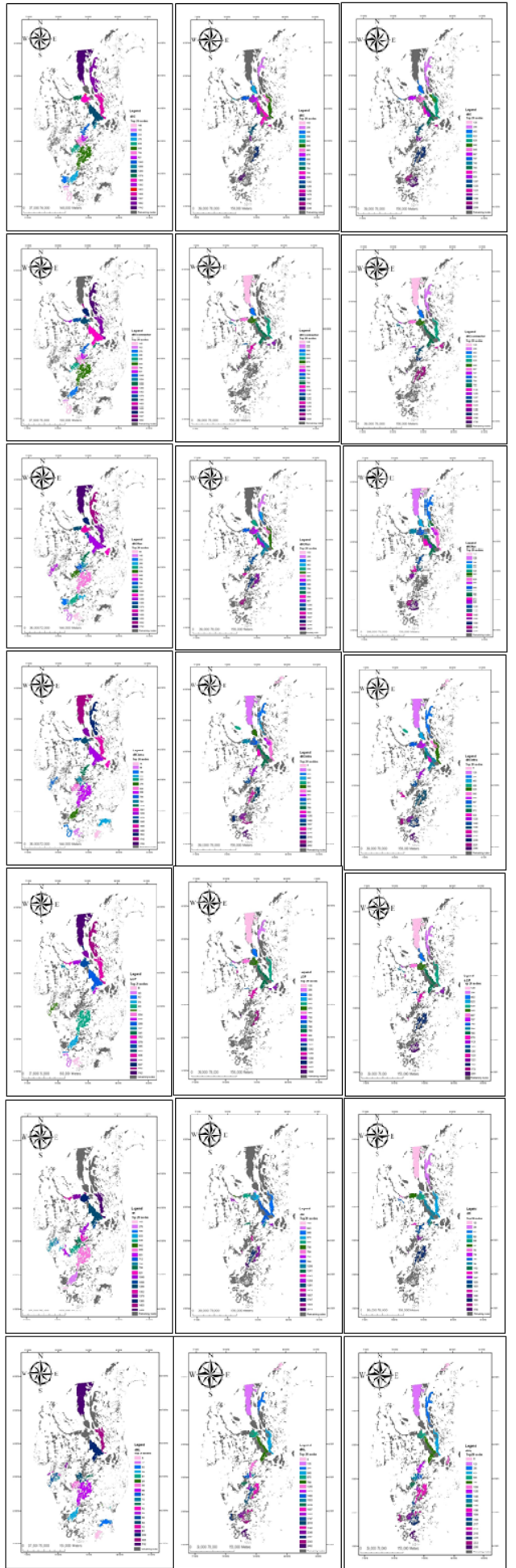

Figure 8: Top 20 imporatnt nodes (forest patch) value for connectivity on the basis of graph theoretic indicies.

Large the patches area are having high dIIc and dLCp value. Components 6 are showing the potential patch for connectivity in Figure 8. From Figure 8 patch node Id namely 1289, 649 are chosen as important patches for connectivity as they have high importance value with reference to $\mathrm{dA}, \mathrm{dIIC}, \mathrm{dLCP}, \mathrm{BC}, \mathrm{dH}$ and dIIC (intra and flux) patch Id 1289, 1385 are chosen as important patch for connectivity with reference dIIC connector. Hence these two patches within the components 6 for connectivity.

\section{Importance of individual habitat patches for landscape connectivity in 1995}

In above given figures shows node importance with reference to area dIIC, dLCP, BC dH, dNL, dA, dIIC (intra, connector, flux) for all the node ID is components 20 at thershold distance $3000 \mathrm{~m}$. Components 20 have 1525 patches (nodes) with total area $19701 \mathrm{~km}^{2}$. The main focus of study is on high importance value indices. Hence consider those patches which participate for connectivity. High important value will facilities the understanding of patch significant for connectivity. Hence top 20 patches (nodes) having a high dA, dIIC, dLCP, BC, dH, dNL, dIIC (intra, connector, flux) value are analyzed as shown in above figures. This approach helped to study the concentration of dIIC value at different intervals. Around 20 patches are getting higher than $1 \mathrm{dIIC}$ value. All remaining patches are in the range $0-1$ Those patches having very big area has high dIIc value s. remaining patches are less than 0.05 .

Since, their area very less node ID (patches) namely 789, and 135 have the highest value dA, dIIC, dIIC intra and flux value. For connecting these two very high important value patches choosing a suitable patch with high to medium important patches required. Fraction dIIc results are shown in Figure. Node ID 135, 789, 649, 206, 1657 got a highest dIIC intra; Node ID 135, 789, 649, 670, 206, got a highest dIIC flux value; Node ID 789, 1242, 1255, 643, 649 got a highest dIIC connector value; Node ID 1255, 789, 1242, 649, 643, 135, got a highest $\mathrm{dH}$ value; Node ID 789, 1255, 1478, 1242, 1574, got a highest BC value; Node Id 789, 1255, 1242, 643, 649, 135 got a highest dLCP value; Node Id 135, 789, 649, 206, 1657, got the highest value of dA. Node IDSs 135 and 789, are shown in graph is got the highest value in all connectivity indices, so that the other node separately in the graph. Patch 165 and 789 got a position under top five in all other patches. Large area patches are having high dIIc and dLCP value in components 20 in shown the potential patch for connectivity. Patch Node ID namely 789,649 , is chosen an important value with reference to dA, dIIC dIC intra flux connector, BC dHND dLCP having the highest important value. In order to connect them patches in their proximity are very important. Hence components 20 cover a central part of the study area. In this region or area the connectivity within component in good and important for movement of species.

\section{Importance of individual habitat patches for landscape connectivity in 1985}

In above given figures showed that important value of connectivity indices with reference to area, dIIC, dA, dLCP, dIIC (intra, flux, connector) $\mathrm{dH}$, and $\mathrm{dNL}$ for all the nodes IDs in components 20 at threshold distances $3000 \mathrm{~m}$. Components 20 has a 1406 nodes (patches) with their total area is $1940 \mathrm{~km}^{2}$. The main focus of the study on the high important patches because considering patch has a high important value will facilitates the understanding of patches significant connectivity. Hence top 20 nodes having a high value of dIIC, dA, dIIC (intra connector, flux) $\mathrm{BC}, \mathrm{dH}, \mathrm{dNL}$ and $\mathrm{dLCP}$ were analyzed these all binary connectivity indices are shown in figures. This approach helped to understand the concentration of different connectivity indices value at different intervals. Around 6 nodes (patches) are getting a higher 
than 20 dIIC value. Remaining patches are less than 20 range 20-0.00005 because there are too small as compared to other dIIC value. dIIC is main indices for connectivity. Rather than BC dH, NL it showed a fewer change of connectivity. Those patches having a big area have a high dIIc value. Node IDs (patches)namely 765 got the highest dIIC, dLCP, dIIC connector and BC value this single node are more important for connectivity of nodes. Node IDs (patches) namely 765, $129,629,1220,107$, and 623 got the highest dIIC value is greater than 20. Node IDs namely 765, 1220, 1207, 623, 629 and 129 got the highest dLCP value is higher than 29 up to the 54 ; Node IDs (patches) namely $1220,765,1207,629,1598$, and 623 got the highest $\mathrm{dH}$ value is higher than 8 . Node IDs (patches) 129, 765, 629 and 206 got the highest dIIC intra value is higher than 8 . Node Id 765, 1207, 623, 1220, and 629 got the highest dIIC connector value id higher than 15 . Those nodes has a high dIIC connector value it helped to connect to two nodes is like a connector between nodes. Node IDs namely 129, 206, 756, 629, and 1598 got the highest dA value is higher than 2. Node IDs namely 129, 765,629 , and 206 got the highest dIIC flux.

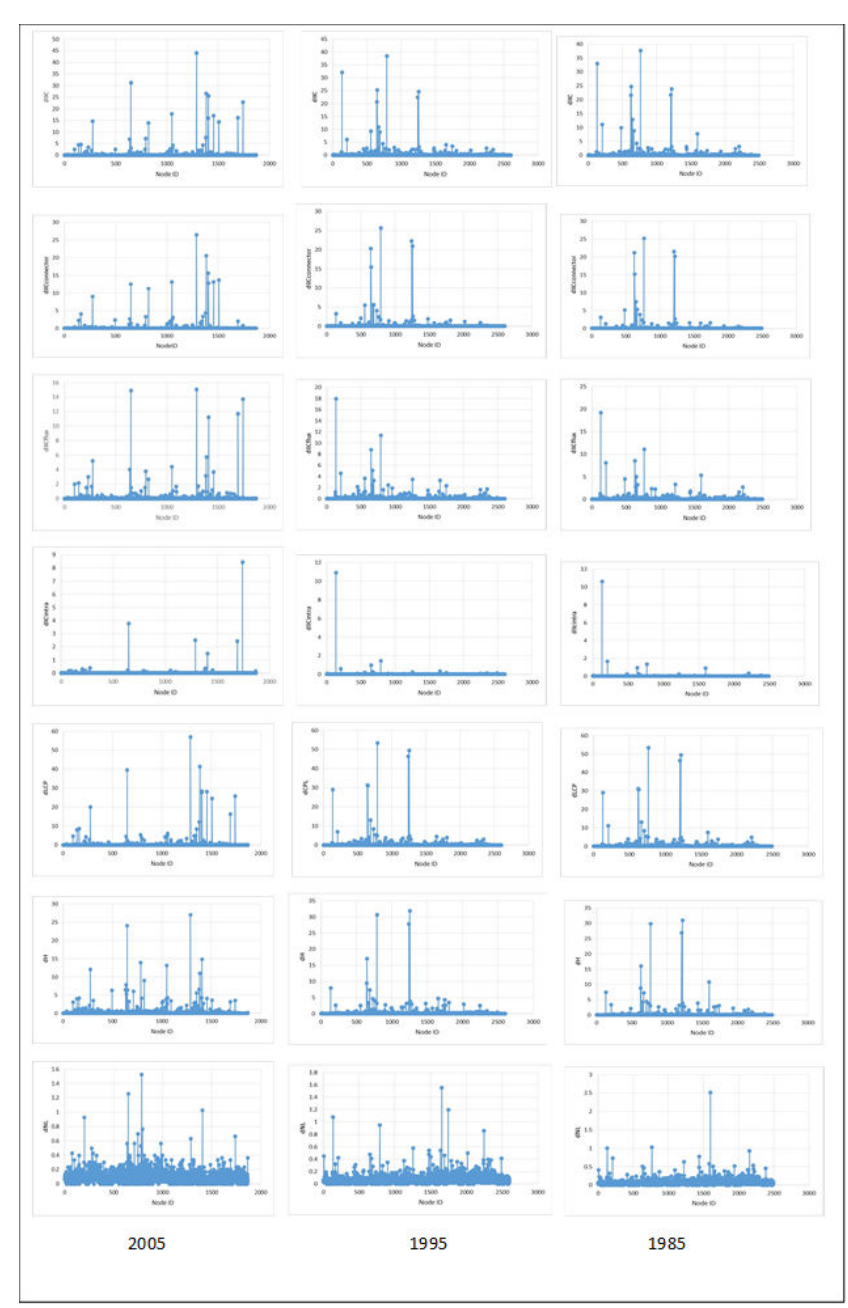

Figure 9: Graph diagram of all nodes value of graph theory indices.

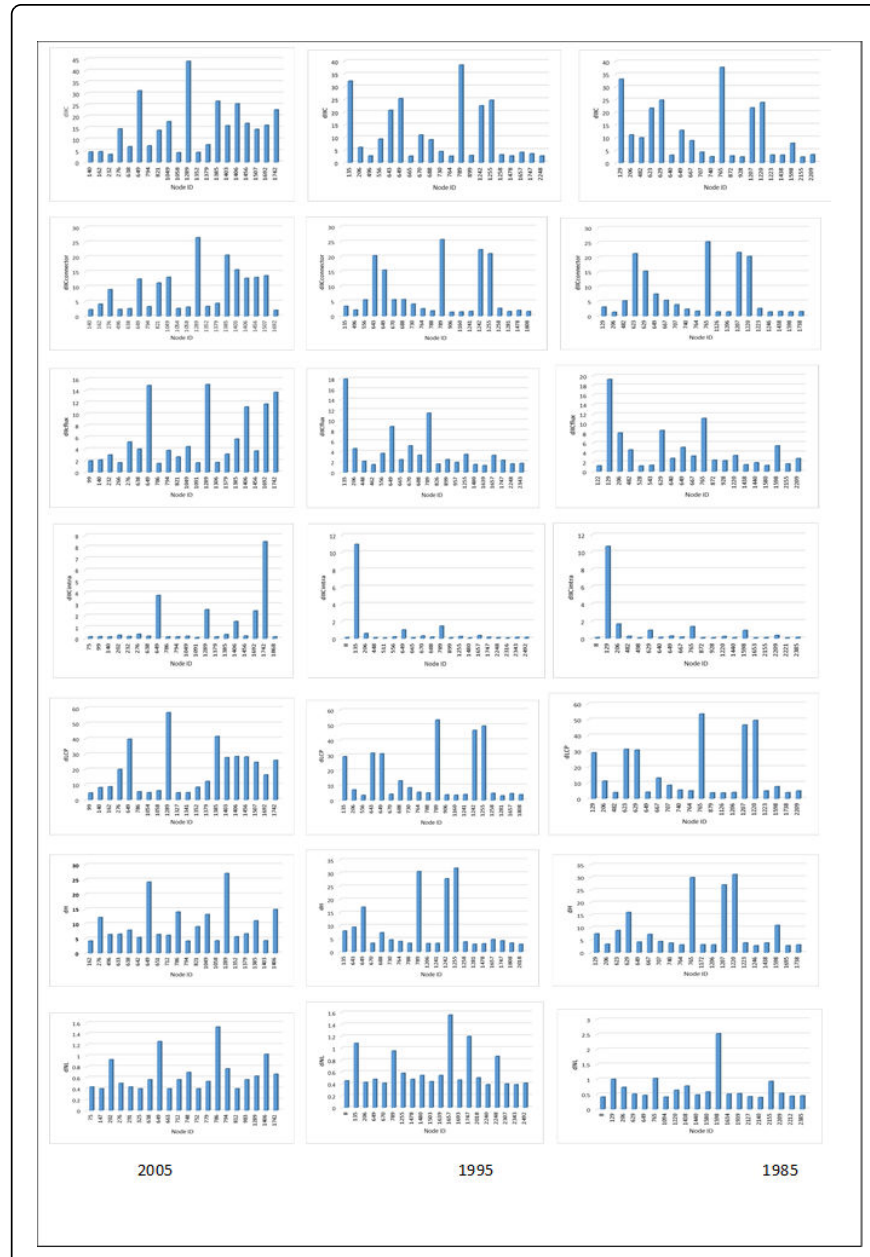

Figure 10: Top 20 Imporatnt nodes (forest patch) value for connectivity on the basis of graph theoretic indicies.

\section{Forest patch connectivity trends in the pennar river basin}

The trend in forest connectivity in the pennar river basin from 1985 to 2005 time period for species dispersal at $3000 \mathrm{~m}$ threshold distance figure shows how much and where forest patches connectivity within the components. Increase the number of nodes from 1985 to 1995 (number of node 2493 to 2602) but decreases from 1995 to 2005 (number of node 2602 to 1870). Figure 9 shows that total area of all node present in the landscape in 2005 the area was calculated is 30532 $\mathrm{km}^{2}$, in 1995 area is $26889 \mathrm{~km}^{2}$, in 1985 area is $2493 \mathrm{~km}^{2}$. This result shows that the small nodes are make large area node within landscape from 1985 to 2005. This occurred when new forest area were planted from other woodlands or only enlarge an existing forest patch. In 2005 pennar basin has a large number of big area nodes were present, due to this it got the highest value of graph theoretic indices. The binary connection model in 2005 year has got a highest connectivity between among the nodes (forest patches). These findings support the consideration of species dispersal and animal movement. If we compare the graph theoretic indices value from 1985 to 2005 time period. dLCP got the highest value in 2005 and dIIC also got the highest value in 2005 its show in Figure 10. we select only dLCP and dIIC for connectivity in different time period. In group of graph theory 
indices fewer changes were detected by simple binary indices (NL, NC $\mathrm{dH}, \mathrm{BC}$ ) and dIIC and dLCP are more complex binary indices (Table 1). There is strong relationship between the score for dIIC and dLCP indices. Thus 2005 has got the important patch for connectivity among the nodes. The forest patch connectivity increases from 1985 to 2005(Figure 11).

\begin{tabular}{|l|l|l|l|l|l|l|}
\hline Indices & Node ID & $\mathbf{1 9 8 5}$ & Node ID & $\mathbf{1 9 9 5}$ & Node ID & $\mathbf{2 0 0 5}$ \\
\hline $\mathrm{dH}$ & 1220 & 31.0623 & 1255 & 31.8701 & 1289 & 27.0372 \\
\hline dIIC & 129 & 32.9892 & 789 & 38.4638 & 1289 & 44.0592 \\
\hline dLCP & 765 & 53.4553 & 789 & 53.3929 & 1289 & 57.0478 \\
\hline dIICC & 765 & 25.2047 & 789 & 25.6354 & 1289 & 26.4914 \\
\hline dIIC flux & 129 & 19.2459 & 135 & 17.9323 & 1289 & 15.0489 \\
\hline dIICintra & 129 & 10.6404 & 789 & 38.4638 & 1724 & 8.44706 \\
\hline dA & 129 & 9.60682 & 135 & 9.4636 & 1742 & 9.66951 \\
\hline dNL & 1598 & 2.52077 & 1657 & 1.55567 & 786 & 1.52368 \\
\hline BC & 765 & 0.17463 & 789 & 0.19066 & 1049 & 0.07258 \\
\hline
\end{tabular}

Table 1: Highest value of binary indices at $3000 \mathrm{~m}$ threshold distance.

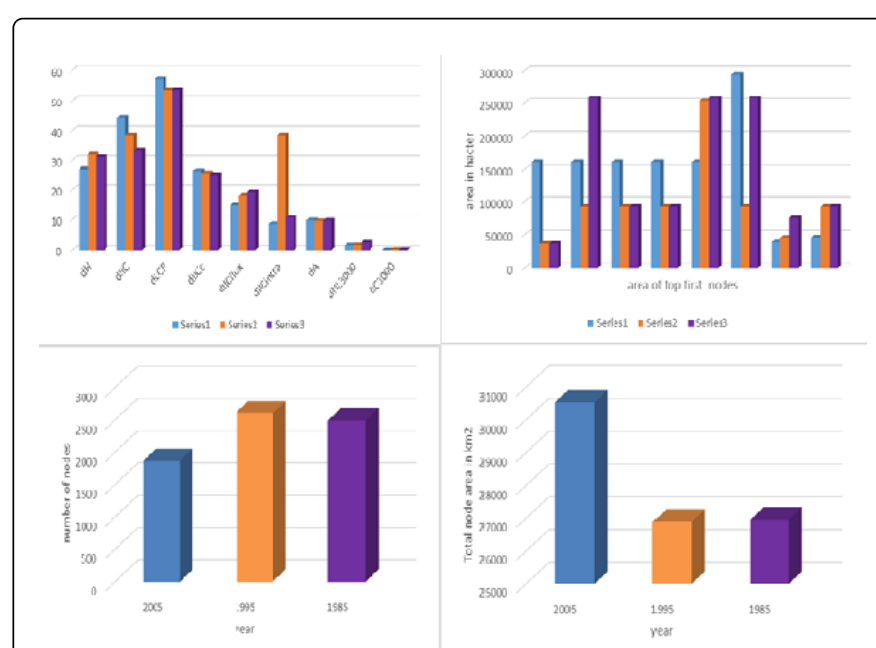

Figure 11: Highest value of binary indices at $3000 \mathrm{~m}$ threshold distance and show the number of node with area in $\mathrm{km}^{2}$ from 2005 to 1985 time period.

\section{Conclusion}

Habitat patches have different roles within the landscape network. They not for serve as site for shelter, for aging and breeding but also produce (or receive) dispersal fluxes to other habitat patches and function as stepping stone that, even when are not the final destination. This study to have a rely on three years spatial data set to asses habitat (forest patch) connectivity. The most important requirement for connectivity is the threshold distance NL and NC. This depends on the number, size and spatial distribution pattern of the forest patches. In this study the very small area patches are more fragment and those patchs has a large area they play vital role in connectivity. when increases the distance the number of links increase and number of components decreases and ultimately the entire landscape show a single component. NL and NC required optimal threshold distance of $3000 \mathrm{~m}$. When the total landscape study area considers. In $3000 \mathrm{~m}$ threshold distance 280 components in 2005, 215 components in 1995, 213 components in 1985.

The increases in component from 1985 to 2005 the gap between year is one decades. In 2005 has a more components because it has a more number of forest patch as compared to both $(1995,1985)$. The spatial distribution of number of patches and area of the different components formed at $3000 \mathrm{~m}$ threshold distance. For further analysis to isolate the top 10 components in different year and to study the connectivity within the components. The spatial distribution components showed that components names 6, 20, 20 (2005, 1995, $1985)$ are most important component within the whole landscape. In 2005 component 6 has 715 nodes (forest patches) and area $22449 \mathrm{~km}^{2}$. In 1995 Component 20 has 1525 node (forest patches) and area is $19701 \mathrm{~km}^{2}$. In 1985 Component 20 has 1406 nodes and area is 19640 $\mathrm{km}^{2}$. In 2005 had a less number of node as compared to 1985 or 1995 but the area of the node is very large due this connectivity within the components is found to more optimal. Therefore, connectivity among the patches within this component $(6,20$, and 20$)$ is found to be more important effort than connecting other components in whole landscape.

The IIC indices provided all properties of perfect index based on the given parameters and condition. It is very sensitive to all kind of negative changes that can affect the habitat mosaic. It helped detected which more changes are more critical for conservation. LCP and IIC importance value was almost invariable to changing scale [21] thus the landscape coincidence probability and integral index connectivity play an important role in finding of potential nodes for biodiversity conservation in applying the IIC more focus is on the topological reachability of the network and less focus on the actual quantities of organism that flow throughout the landscape [25].

By using graph theory for connectivity of forest patch within the components and whole landscape the important thing is that first finding out the threshold distance for connectivity among the patches through the study of component numbers, number of links, dIIC and dLCP it easy to identify the potential patches for connectivity. Graph theory is essential tool for connectivity of forest in the landscape connectivity [19]. The graph theory approach in other many revalent discipline. And make a modeling methods. It is heuristic framework which can be used with very little data and improved from the primary results [3]. If we identifying the important patches in the landscape through graph theory approach in order to enhance the connectivity between patches. Even if the connectivity between patches is not possible, at increases the patch size can be made on the basis of important value. Theoretical analysis and practical application for improvement of patches is really multifaceted. Complexities are normally due to the outcome of collective ensembles of many entities, not as a result of the complexity of interactions. Analysis with reference to inter-component and the intra-component will enhance the study of the specific habitat for biodiversity conservation.

The graph theory approach for ecological modeling with habitat forest patches within landscape. The connectivity indices dIIC, dLCP, $\mathrm{dH}$, and $\mathrm{BC}$ are based on the graph theory provided adequate understanding of patch efficiency for connectivity of habitat patch for the movement of animals, seed dispersal, and exchange genetic material. Fewer changes were detected by the simple binary indices 
(NL, NC, dH). In pennar river basin the central part from north to south in this region important patches are there, they got a highest value of connectivity indices. And also have a very large area. So that its area can be increases in order to connect with other patches. BC measure the single node connectivity it shows the which node is more important in the landscape. $\mathrm{H}$ index it finds the shortest topological distance between two nodes it very helped for finding shortest distance for movement animals. Thus, dIIC important value along with fractions (dIICintra, dIICconnector, dIICflux). dIIC helped for identify the potential habitat patches for biodiversity and conservation. dIIC and dLCP are important binary indices for identifying the important patches in the landscape of the entire study because these are complex binary indices. While NL, NC, H, BC are simple binary indices for which fewer change can be detected. In 2005 has got the highest value of indices at $3000 \mathrm{~m}$ threshold. Thus, on the basis of graph theory indices value in 2005 the connectivity more as compared to the in 1995 or 1985 year.

Limitation of Conefor sensinode software is that the processing is very slow. Especially obtaining the nodes and distance from arc GIS extension take many hours. Therefore, first choosing the necessary patches foe analysis will serve the output quickly and result will be efficient this technique is better for forest conservation and biodiversity conservation.

\section{References}

1. Haray F (1969) Graph theory. London: Addition Wesley. Reading Mass.

2. Keitt T, Urban D, Milne B (1997) Detecting critical scales in fragmented landscapes. Conservation Ecology 1:4

3. Bunn AG, Urban DL, Keitt TH (2000) Landscape connectivity: a conservation application of graph theory. Journal of Environmental Management 59: 265-278.

4. García-Feced C, Saura S, Elena-Rosselló R (2011) Improving landscape connectivity in forest districts: a two-stage process for prioritizing agricultural patches for reforestation. Forest Ecology and Management 261: 154-161.

5. Urban D, Keitt $\mathrm{T}$ (2001) Landscape connectivity: a graph-theoretic perspective. Ecology 82: 1205-1218.

6. Zetterberg A, Mörtberg UM, Balfors B (2010) Making graph theory operational for landscape ecological assessments, planning, and design. Landscape and Urban Planning 95: 181-191.

7. Saura S, Rubio L (2010) A common currency for the different ways in which patches and links can contribute to habitat availability and connectivity in the landscape. Ecography 33: 523-537.

8. Albert R, Barabási AL (2002) Statistical mechanics of complex networks. Reviews of Modern Physics 74: 47.

9. Melián CJ, Bascompte J (2002) Complex networks: two ways to be robust? Ecology Letters 5: 705-708.
10. Gastner MT, Newman ME (2006) The spatial structure of networks. The European Physical Journal B-Condensed Matter and Complex Systems 49: 247-252.

11. Fortuna MA, Garcia C, Guimarães Jr PR, Bascompte J (2008) Spatial mating networks in insect-pollinated plants. Ecology Letters 11: 490-498.

12. Fuller T, Munguía M, Mayfield M, Sánchez-Cordero V, Sarkar S (2006) Incorporating connectivity into conservation planning: a multi-criteria case study from central Mexico. Biological Conservation 133: 131-142.

13. Adriaensen F, Chardon JP, De Blust G, Swinnen E, Villalba S, et al. (2003) The application of 'least-cost' modelling as a functional landscape model. Landscape and Urban Planning 64: 233-247.

14. Drielsma M, Manion G, Ferrier S (2007) The spatial links tool: automated mapping of habitat linkages in variegated landscapes. Ecological Modelling 200: 403-411.

15. McRae BH, Dickson BG, Keitt TH, Shah VB (2008) Using circuit theory to model connectivity in ecology, evolution, and conservation. Ecology 89: 2712-2724.

16. Pinto N, Keitt TH (2009) Beyond the least-cost path: evaluating corridor redundancy using a graph-theoretic approach. Landscape Ecology 24: 253-266.

17. Minor ES, Urban DL (2007) Graph theory as a proxy for spatially explicit population models in conservation planning. Ecological Applications 17: 1771-1782.

18. Moilanen A, Nieminen M (2002) Simple connectivity measures in spatial ecology. Ecology 83: 1131-1145.

19. Pascual-Hortal L, Saura S (2008) Integrating landscape connectivity in broad-scale forest planning through a new graph based habitat availability methodology: application to capercaillie (Tetraourogallus) in Catalonia (NE Spain). European Journal of Forest Research 127: 23-31.

20. Kramer-Schadt S, Revilla E, Wiegand T, Breitenmoser URS (2004) Fragmented landscapes, road mortality and patch connectivity: modelling influences on the dispersal of Eurasian lynx. Journal of Applied Ecology 41: 711-723.

21. Pascual-Hortal L, Saura S (2007) Impact of spatial scale on the identification of critical habitat patches for the maintenance of landscape connectivity. Landscape and Urban Planning 83: 176-186.

22. Jaeger JA (2000) Landscape division, splitting index, and effective mesh size: new measures of landscape fragmentation. Landscape Ecology 15: 115-130.

23. Saura S, Torne J (2009) Conefor Sensinode 2.2: a software package for quantifying the importance of habitat patches for landscape connectivity. Environmental Modelling \& Software 24: 135-139.

24. Pascual-Hortal L, Saura S (2006) Comparison and development of new graph-based landscape connectivity indices: towards the priorization of habitat patches and corridors for conservation. Landscape Ecology 21: 959-967.

25. Bodin O, Saura S (2010) Ranking individual habitat patches as connectivity providers: integrating network analysis and patch removal experiments. Ecological Modelling 221: 2393-2405. 